\title{
ON THE DYNAMICS OF A RATIONAL DIFFERENCE EQUATION
}

\author{
A. M. AHMED and IBRAHIM A. AHMED \\ Department of Mathematics \\ Faculty of Science \\ Al-Azhar University \\ Nasr City (11884), \\ Cairo, Egypt \\ e-mail: ahmedelkb@yahoo.com \\ Department of Basic Science \\ Faculty of Information System and Computer Science \\ October 6 University \\ Giza, Egypt \\ e-mail: i_hakim84@yahoo.com
}

\begin{abstract}
In this paper, we investigate the global behaviour of the difference equation

$$
x_{n+1}=\frac{\alpha x_{n-1}}{\beta+\gamma x_{n-2}^{p} x_{n-4}+\gamma x_{n-2} x_{n-4}^{p}}, \quad n=0,1,2, \ldots,
$$

with positive parameters and non-negative initial conditions.
\end{abstract}

\section{Introduction}

Difference equations appear as natural descriptions of observed evolution phenomena because most measurements of time evolving 2010 Mathematics Subject Classification: 39A10.

Keywords and phrases: recursive sequences, global asymptotic stability, oscillation, period two solutions, semicycles.

Received May 22, 2017; Revised July 7, 2017

(C) 2017 Scientific Advances Publishers 
variables are discrete and as such these equations are in their own right important mathematical models. More importantly, difference equations also appear in the study of discretization methods for differential equations. Several results in the theory of difference equations have been obtained as more or less natural discrete analogues of corresponding results of differential equations.

Recently, there has been a lot of interest in studying the global attractivity, boundedness character, periodicity and the solution form of nonlinear difference equations.

El-Owaidy et al. [1] investigated the global asymptotic behaviour and the periodic character of the solutions of the difference equation

$$
x_{n+1}=\frac{\alpha x_{n-1}}{\beta+\gamma x_{n-2}^{p}}, \quad n=0,1,2, \ldots,
$$

where the parameters $\alpha, \beta, \gamma$, and $p$ are non-negative real numbers.

Other related results on rational difference equations can be found in [2-8].

In this paper, we investigate the global asymptotic behaviour and the periodic character of the solutions of the fifth-order difference equation

$$
x_{n+1}=\frac{\alpha x_{n-1}}{\beta+\gamma x_{n-2}^{p} x_{n-4}+\gamma x_{n-2} x_{n-4}^{p}}, \quad n=0,1,2, \ldots,
$$

where the parameters $\alpha, \beta, \gamma$, and $p$ are positive real numbers and the initial conditions $x_{-4}, x_{-3}, x_{-2}, x_{-1}$, and $x_{0}$ are non-negative real numbers.

We generalize the results due to Erdogan et al. [8] corresponding to the difference equation

$$
x_{n+1}=\frac{\alpha x_{n-1}}{\beta+\gamma x_{n-2}^{2} x_{n-4}+\gamma x_{n-2} x_{n-4}^{2}}, \quad n=0,1,2, \ldots,
$$


where the parameters $\alpha, \beta$, and $\gamma$ are positive real numbers and the initial conditions $x_{-4}, x_{-3}, x_{-2}, x_{-1}$, and $x_{0}$ are non-negative real numbers.

They proved the following results:

Theorem 1.1. (i) If $r<1$, then the equilibrium point $\overline{y_{1}}=0$ of Equation (1.2) is global asymptotically stable.

(ii) If $r>1$, then the equilibrium point $\overline{y_{1}}=0$ of Equation (1.2) is a saddle point.

(iii) When $r>1$, then the positive equilibrium point $\overline{y_{2}}=\left(\frac{r-1}{2}\right)^{\frac{1}{p+1}}$ of Equation (1.2) is unstable.

Theorem 1.2. Assume that $r=1$, then Equation (1.2) possesses the prime period two solution

$$
\ldots, \phi, 0, \phi, 0, \ldots
$$

with $\phi>0$. Furthermore, every solution of Equation (1.2) converges to a period two solution (1.3) with $\phi \geq 0$.

Theorem 1.3. Assume that $r>1$, then Equation (1.2) possesses an unbounded solution.

We need the following definitions:

Definition 1. Let $I$ be an interval of real numbers and let $f: I^{k+1} \rightarrow I$ be a continuously differentiable function. Consider the difference equation

$$
x_{n+1}=f\left(x_{n}, x_{n-1}, \ldots, x_{n-k}\right), \quad n=0,1, \ldots,
$$

with $x_{-k}, x_{-k+1}, \ldots, x_{0} \in I$. Let $\bar{x}$ be the equilibrium point of Equation (1.4). The linearized equation of Equation (1.4) about the equilibrium point $\bar{x}$ is 


$$
y_{n+1}=c_{1} y_{n}+c_{2} y_{n-1}+\ldots+c_{k+1} y_{n-k},
$$

where $c_{1}=\frac{\partial f}{\partial x_{n}}(\bar{x}, \bar{x}, \ldots, \bar{x}), c_{2}=\frac{\partial f}{\partial x_{n-1}}(\bar{x}, \bar{x}, \ldots, \bar{x}), \ldots, c_{k+1}=\frac{\partial f}{\partial x_{n-k}}$ $(\bar{x}, \bar{x}, \ldots, \bar{x})$. The characteristic equation of Equation (1.5) is

$$
\lambda^{k+1}-\sum_{i=1}^{k+1} c_{i} \lambda^{k-i+1}=0 .
$$

(i) The equilibrium point $\bar{x}$ of Equation (1.4) is locally stable if for every $\epsilon>0$, there exists $\delta>0$ such that for all $x_{-k}, x_{-k+1}, \ldots, x_{-1}, x_{0} \in I$ with $\left|x_{-k}-\bar{x}\right|+\left|x_{-k+1}-\bar{x}\right|+\ldots+\left|x_{0}-\bar{x}\right|<\delta$, we have $\left|x_{n}-\bar{x}\right|<\epsilon$ for all $n \geq-k$.

(ii) The equilibrium point $\bar{x}$ of Equation (1.4) is locally asymptotically stable if $\bar{x}$ is locally stable and there exists $\gamma>0$, such that for all $x_{-k}, x_{-k+1}, \ldots, x_{-1}, x_{0} \in I$ with $\left|x_{-k}-\bar{x}\right|+\left|x_{-k+1}-\bar{x}\right|+\ldots+\left|x_{0}-\bar{x}\right|<\gamma$, we have $\lim _{n \rightarrow \infty} x_{n}=\bar{x}$.

(iii) The equilibrium point $\bar{x}$ of Equation (1.4) is global attractor if for all $x_{-k}, x_{-k+1}, \ldots, x_{-1}, x_{0} \in I$, we have $\lim _{n \rightarrow \infty} x_{n}=\bar{x}$.

(iv) The equilibrium point $\bar{x}$ of Equation (1.4) is globally asymptotically stable if $\bar{x}$ is locally stable, and $\bar{x}$ is also a global attractor of Equation (1.4).

(v) The equilibrium point $\bar{x}$ of Equation (1.4) is unstable if $\bar{x}$ is not locally stable.

Definition 2. A positive semicycle of $\left\{x_{n}\right\}_{n=-k}^{\infty}$ of Equation (1.4) consists of a 'string' of terms $\left\{x_{l}, x_{l+1}, \ldots, x_{m}\right\}$, all greater than or equal to $\bar{x}$, with $l \geq-k$ and $m<\infty$ and such that either $l=-k$ or $l>-k$ and $x_{l-1}<\bar{x}$ and either $m=\infty$ or $m<\infty$ and $x_{m+1}<\bar{x}$.

A negative semicycle of $\left\{x_{n}\right\}_{n=-k}^{\infty}$ of Equation (1.4) consists of a 'string' of terms $\left\{x_{l}, x_{l+1}, \ldots, x_{m}\right\}$, all less than $\bar{x}$, with $l \geq-k$ and 
$m<\infty$ and such that either $l=-k$ or $l>-k$ and $x_{l-1} \geq \bar{x}$ and either $m=\infty$ or $m<\infty$ and $x_{m+1} \geq \bar{x}$.

Definition 3. A solution $\left\{x_{n}\right\}_{n=-k}^{\infty}$ of Equation (1.4) is called nonoscillatory if there exists $N \geq-k$ such that either $x_{n} \geq \bar{x}, \forall n \geq N$ or $x_{n}<\bar{x}, \forall n \geq N$, and it is called oscillatory if it is not nonoscillatory.

(a) A sequence $\left\{x_{n}\right\}_{n=-k}^{\infty}$ is said to be periodic with period $p$ if

$$
x_{n+p}=x_{n} \text { for all } n \geq-k \text {. }
$$

(b) A sequence $\left\{x_{n}\right\}_{n=-k}^{\infty}$ is said to be periodic with prime period $p$ if it is periodic with period $p$ and $p$ is the least positive integer for which (1.7) holds.

We need the following theorem:

Theorem 1.4. (i) If all roots of Equation (1.6) have absolute value less than one, then the equilibrium point $\bar{x}$ of Equation (1.2) is locally asymptotically stable.

(ii) If at least one of the roots of Equation (1.6) has absolute value greater than one, then $\bar{x}$ is unstable.

The equilibrium point $\bar{x}$ of Equation (1.4) is called a saddle point if Equation (1.6) has roots both inside and outside the unit disk.

\section{Main Results}

In this section, we investigate the dynamics of Equation (1.1) under the assumptions that all parameters in the equation are positive and the initial conditions are non-negative.

The change of variables $x_{n}=\left(\frac{\beta}{\gamma}\right)^{\frac{1}{p+1}} y_{n}$ reduces Equation (1.1) to the difference equation

$$
y_{n+1}=\frac{r y_{n-1}}{1+y_{n-2}^{p} y_{n-4}+y_{n-2} y_{n-4}^{p}}, \quad n=0,1,2, \ldots,
$$


where $r=\frac{\alpha}{\beta}>0$.

Note that $\overline{y_{1}}=0$ is always an equilibrium point of Equation (2.1).

When $r>1$, Equation (2.1) also possesses the unique positive equilibrium $\overline{y_{2}}=\left(\frac{r-1}{2}\right)^{\frac{1}{p+1}}$.

Theorem 2.1. The following statements are true:

(i) If $r<1$, then the equilibrium point $\overline{y_{1}}=0$ of Equation (2.1) is locally asymptotically stable.

(ii) If $r>1$, then the equilibrium point $\overline{y_{1}}=0$ of Equation (2.1) is a saddle point.

(iii) When $r>1$, then the positive equilibrium point $\overline{y_{2}}=\left(\frac{r-1}{2}\right)^{\frac{1}{p+1}}$ of Equation (2.1) is unstable.

Proof. The linearized equation of Equation (2.1) about the equilibrium point $\overline{y_{1}}=0$ is

$$
z_{n+1}=r z_{n-1}, \quad n=0,1,2, \ldots,
$$

so, the characteristic equation of Equation (2.1) about the equilibrium point $\overline{y_{1}}=0$ is

$$
\lambda^{2}-r=0
$$

and hence, the proof of (i) and (ii) follows from Theorem 1.4.

For (iii), we assume that $r>1$, then the linearized equation of Equation (2.1) about the equilibrium point $\overline{y_{2}}=\left(\frac{r-1}{2}\right)^{\frac{1}{p+1}}$ has the form

$$
z_{n+1}=z_{n-1}-\frac{(r-1)(p+1)}{2 r} z_{n-2}-\frac{(r-1)(p+1)}{2 r} z_{n-4}, \quad n=0,1,2, \ldots,
$$


so, the characteristic equation of Equation (2.1) about the equilibrium point $\overline{y_{2}}=\left(\frac{r-1}{2}\right)^{\frac{1}{p+1}}$ is

$$
f(\lambda)=\lambda^{5}-\lambda^{3}+\frac{(r-1)(p+1)}{2 r} \lambda^{2}+\frac{(r-1)(p+1)}{2 r}=0 .
$$

It is clear that $f(\lambda)$ is continuous because it is a polynomial.

$$
f(-1)=(-1)^{5}-(-1)^{3}+\frac{(r-1)(p+1)}{2 r}(-1)^{2}+\frac{(r-1)(p+1)}{2 r}=\frac{(r-1)(p+1)}{r},
$$

which implies that $f(-1)>0$ because $r>1$ and $p$ is positive.

$$
\begin{aligned}
\lim _{\lambda \rightarrow-\infty} f(\lambda) & =\lim _{\lambda \rightarrow-\infty}\left[\lambda^{5}-\lambda^{3}+\frac{(r-1)(p+1)}{2 r} \lambda^{2}+\frac{(r-1)(p+1)}{2 r}\right] \\
& =\lim _{\lambda \rightarrow-\infty}\left[\lambda^{2}\left(\lambda^{3}-\lambda+\frac{(r-1)(p+1)}{2 r}\right)+\frac{(r-1)(p+1)}{2 r}\right] \\
& =\lim _{\lambda \rightarrow-\infty}\left[\lambda^{2}\left(\lambda\left(\lambda^{2}-1\right)+\frac{(r-1)(p+1)}{2 r}\right)+\frac{(r-1)(p+1)}{2 r}\right]=-\infty,
\end{aligned}
$$

which implies that $\lim _{\lambda \rightarrow-\infty} f(\lambda)<0$.

Which implies that $f(\lambda)$ has a root in the interval $(-\infty,-1)$, and so, $\overline{y_{2}}=\left(\frac{r-1}{2}\right)^{\frac{1}{p+1}}$ is an unstable equilibrium point.

This completes the proof.

Theorem 2.2. Assume that $r<1$, then, the equilibrium point $\overline{y_{1}}=0$ of Equation (2.1) is globally asymptotically stable.

Proof. We know by Theorem 2.1 that the equilibrium point $\overline{y_{1}}=0$ of Equation (2.1) is locally asymptotically stable. So, let $\left\{y_{n}\right\}_{n=-4}^{\infty}$ be a solution of Equation (2.1). It suffices to show that

$$
\lim _{n \rightarrow \infty} y_{n}=0 \text {. }
$$


Since

$$
0 \leq y_{n+1}=\frac{r y_{n-1}}{1+y_{n-2}^{p} y_{n-4}+y_{n-2} y_{n-4}^{p}} \leq r y_{n-1}<y_{n-1} .
$$

So, the even terms of this solution decrease to a limit (say $L_{1} \geq 0$ ), and the odd terms decrease to a limit (say $L_{2} \geq 0$ ), which implies that

$$
L_{1}=\frac{r L_{1}}{1+2 L_{2}^{p+1}} \quad \text { and } \quad L_{2}=\frac{r L_{2}}{1+2 L_{1}^{p+1}}
$$

If $L_{1} \neq 0 \Rightarrow L_{2}=\left(\frac{r-1}{2}\right)^{\frac{1}{p+1}}<0$, which is a contradiction, so $L_{1}=0$, which implies that $L_{2}=0$.

So,

$$
\lim _{n \rightarrow \infty} y_{n}=0
$$

which the proof is complete.

Theorem 2.3. Assume that $r=1$, then Equation (2.1) possesses the prime period two solution

$$
\ldots, \phi, 0, \phi, 0, \ldots
$$

with $\phi>0$. Furthermore, every solution of Equation (2.1) converges to a period two solution (2.2) with $\phi \geq 0$.

Proof. Let

$$
\ldots, \phi, \psi, \phi, \psi, \ldots
$$

be period two solutions of Equation (2.1). Then

$$
\phi=\frac{r \phi}{1+2 \psi^{p+1}} \quad \text { and } \quad \psi=\frac{r \psi}{1+2 \phi^{p+1}},
$$

so,

$$
2 \phi \psi=\frac{(r-1)(\phi-\psi)}{\psi^{p}-\phi^{p}},
$$


which implies that $r-1 \leq 0$.

If $r<1$, then this implies that $\phi<0$ or $\phi<0$, which is impossible, so $r=1$.

To complete the proof, assume that $r=1$ and let $\left\{y_{n}\right\}_{n=-4}^{\infty}$ be a solution of Equation (2.1), then

$$
y_{n+1}-y_{n-1}=\frac{-y_{n-1}\left(y_{n-2}^{p} y_{n-4}+y_{n-2} y_{n-4}^{p}\right)}{1+y_{n-2}^{p} y_{n-4}+y_{n-2} y_{n-4}^{p}} \leq 0, \quad n=0,1,2, \ldots
$$

So, the even terms of this solution decrease to a limit (say $\Phi \geq 0$ ), and the odd terms decrease to a limit (say $\Psi \geq 0$ ). Thus,

$$
\Phi=\frac{\Phi}{1+2 \Psi^{p+1}} \quad \text { and } \quad \Psi=\frac{\Psi}{1+2 \Phi^{p+1}}
$$

which implies that

$$
2 \Phi \Psi^{p+1}=0 \quad \text { and } \quad 2 \Phi^{p+1} \Psi=0 .
$$

Then the proof is complete.

Theorem 2.4. Assume that $r>1$, and let $\left\{y_{n}\right\}_{n=-4}^{\infty}$ be a solution of Equation (2.1) such that

$$
y_{-4}, y_{-2}, y_{0} \geq \overline{y_{2}}=\left(\frac{r-1}{2}\right)^{\frac{1}{p+1}} \quad \text { and } \quad y_{-3}, y_{-1}<\overline{y_{2}}=\left(\frac{r-1}{2}\right)^{\frac{1}{p+1}} \text {, }
$$

or

$$
y_{-4}, y_{-2}, y_{0}<\overline{y_{2}}=\left(\frac{r-1}{2}\right)^{\frac{1}{p+1}} \quad \text { and } \quad y_{-3}, y_{-1} \geq \overline{y_{2}}=\left(\frac{r-1}{2}\right)^{\frac{1}{p+1}}
$$

Then $\left\{y_{n}\right\}_{n=-4}^{\infty}$ oscillates about $\overline{y_{2}}=\left(\frac{r-1}{2}\right)^{\frac{1}{p+1}}$ with a semicycle of length one. 
Proof. Assume that (2.3) holds. (The case where (2.4) holds is similar and will be omitted.) Then,

$$
y_{1}=\frac{r y_{-1}}{1+y_{-2}^{p} y_{-4}+y_{-2} y_{-4}^{p}}<\frac{r \overline{y_{2}}}{1+2{\overline{y_{2}}}^{p+1}}=\overline{y_{2}}
$$

and

$$
y_{2}=\frac{r y_{0}}{1+y_{-1}^{p} y_{-3}+y_{-1} y_{-3}^{p}}>\frac{r \overline{y_{2}}}{1+2{\overline{y_{2}}}^{p+1}}=\overline{y_{2}}
$$

and then the proof follows by induction.

Theorem 2.5. Assume that $r>1$, then Equation (2.1) possesses an unbounded solution.

Proof. From Theorem 2.4, we can assume without loss of generality that the solution $\left\{y_{n}\right\}_{n=-4}^{\infty}$ of Equation (2.1) is such that

$$
y_{2 n-1}<\overline{y_{2}}=\left(\frac{r-1}{2}\right)^{\frac{1}{p+1}} \quad \text { and } \quad y_{2 n}>\overline{y_{2}}=\left(\frac{r-1}{2}\right)^{\frac{1}{p+1}}, \quad \text { for } n \geq 0 .
$$

Then

$$
y_{2 n+1}=\frac{r y_{2 n-1}}{1+y_{2 n-2}^{p} y_{2 n-4}+y_{2 n-2} y_{2 n-4}^{p}}<\frac{r y_{2 n-1}}{1+2{\overline{y_{2}}}^{p+1}}=y_{2 n-1},
$$

and

$$
y_{2 n+2}=\frac{r y_{2 n}}{1+y_{2 n-1}^{p} y_{2 n-3}+y_{2 n-1} y_{2 n-3}^{p}}>\frac{r y_{2 n}}{1+2{\overline{y_{2}}}^{p+1}}=y_{2 n},
$$

from which it follows that

$$
\lim _{n \rightarrow \infty} y_{2 n}=\infty \quad \text { and } \quad \lim _{n \rightarrow \infty} y_{2 n+1}=0 .
$$

Then, the proof is complete.

Remark. If $p=2$, the results in [8] follow directly. 


\section{References}

[1] H. M. El-Owaidy, A. M. Ahmed and A. M. Youssef, The dynamics of the recursive sequence $x_{n+1}=\frac{\alpha x_{n-1}}{\beta+\gamma x_{n-2}^{p}}$, Appl. Math. Lett. 18(9) (2005), 1013-1018.

[2] A. M. Ahmed, On the dynamics of a higher order rational difference equation, Discrete Dynamics in Nature and Society 2011, Article ID 419789, 8 pages, doi:10.1155/2011/419789.

[3] A. M. Ahmed, H. M. El-Owaidy, A. E. Hamza and A. M. Youssef, On the recursive sequence $x_{n+1}=\frac{a+b x_{n-1}}{A+B x_{n}^{k}}$, Journal of Applied Mathematics \& Informatics 27(1-2) (2009), 275-289.

[4] C. Cinar, On the positive solutions of the difference equation $x_{n+1}=\frac{x_{n-1}}{1+x_{n} x_{n-1}}$, Appl. Math. Comp. 150 (2004), 21-24.

[5] C. Cinar, On the difference equation $x_{n+1}=\frac{x_{n-1}}{-1+x_{n} x_{n-1}}$, Appl. Math. Comp. 158 (2004), 813-816.

[6] C. Cinar, On the positive solutions of the difference equation $x_{n+1}=\frac{a x_{n-1}}{1+b x_{n} x_{n-1}}$, Appl. Math. Comp. 156 (2004), 587-590.

[7] C. Cinar, R. Karatas and I. Yalcinkaya, On solutions of the difference equation $x_{n+1}=\frac{x_{n-3}}{-1+x_{n} x_{n-1} x_{n-2} x_{n-3}}$, Mathematica Bohemica. 132(3) (2007), 257-261.

[8] M. Emre Erdogan, Cengiz Cinar and I. Yalçınkaya, On the dynamics of the recursive sequence $x_{n+1}=\frac{x_{n-1}}{\beta+\gamma x_{n-2}^{2} x_{n-4}+\gamma x_{n-2} x_{n-4}^{2}}$, Comp. \& Math. Appl. Math. 61 (2011), 533-537. 